\title{
Local growth envelopes of spaces of generalized smoothness: the sub- critical case
}

\author{
António M. Caetano*1 and Susana D. Moura**2 \\ ${ }^{1}$ Departamento de Matemática, Universidade de Aveiro, 3810-193 Aveiro, Portugal \\ 2 Departamento de Matemática, Universidade de Coimbra, Apartado 3008, 3001-454 Coimbra, Portugal
}

Received 8 October 2002, revised 14 April 2003, accepted 22 April 2003

Published online 13 August 2004

Key words Function spaces of generalized smoothness, local growth envelopes, essential unboundedness MSC (2000) Primary: 46E35

The concept of local growth envelope $\left(\mathcal{E}_{\mathrm{LG}} A, u\right)$ of the quasi-normed function space $A$ is applied to the spaces of generalized smoothness $B_{p q}^{(s, \Psi)}\left(\mathbb{R}^{n}\right)$ and $F_{p q}^{(s, \Psi)}\left(\mathbb{R}^{n}\right)$ and it is shown that the influence of the function $\Psi$, which is a fine tuning of the main smoothness parameter $s$, is strong enough in order to show up in the corresponding growth envelopes.

\section{Introduction}

One knows from the Sobolev embedding theorem that, given $k \in \mathbb{N}$ and $1<p<\infty$,

$$
W_{p}^{k} \hookrightarrow L_{\infty} \text { if, and only if, } k>\frac{n}{p},
$$

a result which has been generalized to the context of Besov and Triebel-Lizorkin spaces:

With the understanding that $s \in \mathbb{R}$ and $0<p, q \leq \infty$ (and $p \neq \infty$ in the case of the $F$-spaces),

$$
\begin{aligned}
F_{p q}^{s} \hookrightarrow L_{\infty} \text { if, and only if, }\left\{\begin{array}{c}
s>\frac{n}{p} \\
\text { or } \\
s=\frac{n}{p} \text { and } 0<p \leq 1,
\end{array}\right. \\
B_{p q}^{s} \hookrightarrow L_{\infty} \text { if, and only if, }\left\{\begin{array}{c}
s>\frac{n}{p} \\
\text { or } \\
s=\frac{n}{p} \text { and } 0<q \leq 1
\end{array}\right.
\end{aligned}
$$

(cf., for example, [20, Thm. 3.3.1, p. 113], also for reference of people who contributed to this result).

Note that the embeddings in $L_{\infty}$ imply the essential boundedness of the functions involved.

Recently, Haroske [8] and Triebel [25] studied the type of essential unboundedness of functions in such spaces which are not embedded in $L_{\infty}$, and with the further restriction $s>n(1 / p-1)_{+}$for the parameters (basically this guarantees one is dealing with regular distributions). The unboundedness was measured by the determination of the so-called local growth envelope function $\mathcal{E}_{\mathrm{LG}} F_{p q}^{s}$ or $\mathcal{E}_{\mathrm{LG}} B_{p q}^{s}$ (which we shall write as $\mathcal{E}_{\mathrm{LG}} A_{p q}^{s}$, for short), which is a (preferably) continuous representative in the equivalence class of all positive decreasing functions which are equivalent to

$$
\mathcal{E}_{\mathrm{LG}} \mid A_{p q}^{s}(t):=\sup \left\{f^{*}(t):\left\|f \mid A_{p q}^{s}\right\| \leq 1\right\}
$$

\footnotetext{
* Corresponding author: e-mail: acaetano@mat.ua.pt, Phone: +351 234370359, Fax: +351 234382014

** e-mail: smpsd@mat.uc.pt, Phone: +351 239791150, Fax: +351 239832568
} 
in some interval $(0, \varepsilon]$ (for some $\varepsilon \in(0,1])$.

They studied both the sub-critical case (corresponding to $s<n / p$ ) and the critical case (corresponding to $s=n / p$ and either $p>1$ for the $F$-spaces or $q>1$ for the $B$-spaces). In the former case (which is what concerns us here in this paper) they obtained that $\mathcal{E}_{\mathrm{LG}} A_{p q}^{s}$ can be taken to be equal to $t^{-1 / r}$ in some interval of the type $(0, \varepsilon]$, as above, where $r>1$ is such that $s-n / p=-n / r$.

Their results are even better than this. They measured the behaviour of an individual $f^{*}$ against $\mathcal{E}_{\mathrm{LG}} A_{p q}^{s}$ and a measure built on this: the Borel measure $\mu$ associated with $-\log \mathcal{E}_{\mathrm{LG}} A_{p q}^{s}$ on some $(0, \varepsilon]$. They looked for the best exponent $v$ such that

$$
\left(\int_{0}^{\varepsilon}\left(\frac{f^{*}(t)}{\mathcal{E}_{\mathrm{LG}} A_{p q}^{s}(t)}\right)^{v} \mu(d t)\right)^{1 / v} \leq c\left\|f \mid A_{p q}^{s}\right\|
$$

for some constant $c=c(v)$ and all $f \in A_{p q}^{s}$, and they have found that it exists and equals $q$ when $A=B$ and equals $p$ when $A=F$. Actually, since $\mu(d t)$ can be taken to be $d t / t$ in the case of the spaces $A_{p q}^{s},(1.1)$ can also be written as

$$
\left(\int_{0}^{\varepsilon}\left(t^{1 / r} f^{*}(t)\right)^{v} \frac{d t}{t}\right)^{1 / v} \leq c\left\|f \mid A_{p q}^{s}\right\|
$$

so that the results obtained can also be seen as extensions or complements to contributions of many other authors: Peetre [19], Strichartz [21], Herz [9], Brudnyi [1], Goldman [5, 6], Lizorkin [14], Kalyabin and Lizorkin [11], Netrusov [17, 18] and Kolyada [12]. Further detailed references can be found in [24, Rmk. 2.7] and [25, Sect. 15].

Our aim here is to deal with this type of issues but now for the spaces $B_{p q}^{(s, \Psi)}$ and $F_{p q}^{(s, \Psi)}$ of generalized smoothness. There are several approaches, of different degrees of generality, to define such spaces (cf. [11], [15], [2], [13], [3]). We follow here the approach used by Edmunds and Triebel in [3, 4] (cf. also [16]).

We get results of type (1.1) with $A_{p q}^{(s, \Psi)}$ instead of $A_{p q}^{s}$, where $\mathcal{E}_{\mathrm{LG}} A_{p q}^{(s, \Psi)}(t)$ is equivalent to $t^{-1 / r} \Psi(t)^{-1}$ near 0 . The corresponding translation to the formulation of type (1.2) means that on the left-hand side one should have $t^{1 / r} \Psi(t)$ instead of $t^{1 / r}$. For precise formulations, please refer to Section 4 below.

In the case $A=B$ and $p \geq 1$ our results, read in terms of embeddings, are connected with the work of Goldman. In this particular case it would be possible to fit in the general setting considered in [6] and hence obtain necessary and sufficient conditions for having an embedding into a general symmetric space. As a starting point this information would be of no use for us, since it gives no way of measuring the optimality of the target space that can be taken. Following our approach we arrived in a natural way to such a target space and then it would be possible, using Hardy type inequalities developed in [7], to check that the corresponding conditions in [6] are indeed fulfilled.

In the third section we present a lifting property for the spaces $A_{p q}^{(s, \Psi)}$ which might have some interest independently of the main topic under consideration in this paper.

\section{Definitions and first properties}

As usual, $\mathbb{R}^{n}$ denotes the $n$-dimensional real Euclidean space, $\mathbb{N}$ the collection of all natural numbers and $\mathbb{N}_{0}=\mathbb{N} \cup\{0\}$. We use the equivalence $\sim$ in

$$
a_{k} \sim b_{k} \quad \text { or } \varphi(x) \sim \psi(x)
$$

always to mean that there are two positive numbers $c_{1}$ and $c_{2}$ such that

$$
c_{1} a_{k} \leq b_{k} \leq c_{2} a_{k} \text { or } \quad c_{1} \varphi(x) \leq \psi(x) \leq c_{2} \varphi(x)
$$

for all admitted values of the discrete variable $k$ or the continuous variable $x$, where $\left(a_{k}\right)_{k},\left(b_{k}\right)_{k}$ are non-negative sequences and $\varphi, \psi$ are non-negative functions. In the latter case we also write $\varphi \sim_{\text {LG }} \psi$ to mean that $\varphi \sim \psi$ in the smallest of the domains of $\varphi$ and $\psi$, where these functions are further assumed to be decreasing, positive and have domains of the type $(0, \varepsilon]$, for possibly different values of $\varepsilon \in(0,1]$. It is easily seen that $\sim_{L G}$ is an equivalence relation in the family $\mathcal{E}_{\mathrm{LG}}$ of such functions. 
Given two quasi-Banach spaces $X$ and $Y$, we write $X \hookrightarrow Y$ if $X \subset Y$ and the natural embedding of $X$ in $Y$ is continuous.

All unimportant positive constants will be denoted by $c$, occasionally with additional subscripts within the same formula. In what follows log is always taken with respect to base 2 .

Since we do not deal with function spaces on domains different from $\mathbb{R}^{n}$, we always omit the " $\mathbb{R}^{n}$ " in their notation.

\subsection{Special functions}

Definition 2.1 A positive monotone function $\Psi$ on the interval $(0,1]$ is called admissible if

$$
\Psi\left(2^{-j}\right) \sim \Psi\left(2^{-2 j}\right), \quad j \in \mathbb{N}_{0} .
$$

Example 2.2 If $c \in(0,1)$ and $b \in \mathbb{R}$, then

$$
\Psi(x)=|\log c x|^{b}, \quad x \in(0,1],
$$

is an admissible function.

In the following lemma we present some properties of admissible functions that will be useful in the sequel. For further properties we refer to Proposition 1.4 in [16].

Lemma 2.3 Let $\Psi$ be an admissible function according to Definition 2.1.

(i) There exist constants $b \geq 0, c_{1}, c_{2}>0$ such that

$$
c_{1}(1+|\log t|)^{-b} \leq \inf _{0<s \leq 1} \frac{\Psi(t s)}{\Psi(s)} \leq \sup _{0<s \leq 1} \frac{\Psi(t s)}{\Psi(s)} \leq c_{2}(1+|\log t|)^{b},
$$

for any $t \in(0,1]$.

(ii) For any $a, d>0$, there is $\delta>0$ such that

$$
\Psi\left(a t^{d}\right) \sim \Psi(t), \quad t \in(0, \delta) .
$$

Proof. Step 1. In this step we prove (i). Let us assume that $\Psi$ is monotone increasing. Then clearly

$$
\sup _{0<s \leq 1} \frac{\Psi(t s)}{\Psi(s)} \leq 1, \quad t \in(0,1]
$$

By the definition of admissible function and having in mind the assumption made on the monotonicity of $\Psi$, there is a constant $b \geq 0$ such that

$$
\Psi\left(2^{-2 j}\right) \leq \Psi\left(2^{-j}\right) \leq 2^{b k} \Psi\left(2^{-2^{k} j}\right), \quad j \in \mathbb{N}_{0}, k \in \mathbb{N}
$$

from which it follows, by standard arguments, that

$$
\frac{\Psi(t s)}{\Psi(s)} \geq c(1+|\log t|)^{-b}, \quad t \in(0,1]
$$

hence the lower bound in (i).

If the admissible function $\Psi$ is monotone decreasing, then $\Psi^{-1}$ is an increasing admissible function and we can reduce to the case just proved.

Step 2. Here we prove (ii). We may assume without loss of generality that $\Psi$ is monotone increasing. By Proposition 1.4 (iv) of [16], for any $a>0$ there is $j_{0} \in \mathbb{N}$ such that

$$
\Psi\left(a 2^{-j}\right) \sim \Psi\left(2^{-j}\right), \quad j \geq j_{0} .
$$

It then follows, by standard arguments, that

$$
\Psi(a t) \sim \Psi(t), \quad t \in(0, \delta),
$$


for some $\delta \in(0,1)$. Analogously, but using the relation

$$
\Psi\left(2^{-a j}\right) \sim \Psi\left(2^{-j}\right), \quad j \geq j_{0},
$$

instead of (2.3), which is also stated in [16], we can show that

$$
\Psi\left(t^{a}\right) \sim \Psi(t), \quad t \in(0, \delta),
$$

for some $\delta \in(0,1)$. Then (ii) follows from (2.4) and (2.5).

In the sequel we will be concerned with the interpolation with a function parameter built in terms of admissible functions, so that we need to recall some facts and notation about the former and show how to go from the latter to appropriate function parameters.

Definition 2.4 A function $g:(0, \infty) \rightarrow(0, \infty)$ belongs to the class $\mathcal{B}$ if it is continuous, $g(1)=1$ and

$$
\bar{g}(t):=\sup _{s>0} \frac{g(t s)}{g(s)}<\infty, \quad t \in(0, \infty) .
$$

For such a function $g$, the Boyd upper and lower indices $\alpha_{\bar{g}}$ and $\beta_{\bar{g}}$ of $\bar{g}$ are well defined by

$$
\alpha_{\bar{g}}:=\lim _{t \rightarrow+\infty} \frac{\log \bar{g}(t)}{\log t} \quad \text { and } \quad \beta_{\bar{g}}:=\lim _{t \rightarrow 0} \frac{\log \bar{g}(t)}{\log t} .
$$

Lemma 2.5 Let $\Psi$ be a continuous admissible function with $\Psi(1)=1$ and $\varrho:(0, \infty) \rightarrow(0, \infty)$ the function defined by

$$
\varrho(t)= \begin{cases}\Psi(t), & 0<t \leq 1 \\ \Psi\left(t^{-1}\right)^{-1}, & 1 \leq t<\infty .\end{cases}
$$

Then $\varrho \in \mathcal{B}$. Moreover, there exist constants $b \geq 0, c_{1}, c_{2}>0$, only depending on $\Psi$, such that

$$
c_{1}(1+|\log t|)^{-b} \leq \bar{\varrho}(t) \leq c_{2}(1+|\log t|)^{b}, \quad t \in(0, \infty) .
$$

Proof. It is obvious that $\varrho$ is a positive continuous function on $(0, \infty)$ with $\varrho(1)=1$. Thus, to show that $\varrho$ belongs to the class $\mathcal{B}$ it remains to prove (2.6), but this will be the case if we prove (2.8).

We should consider the cases $0<t \leq 1$ and $1 \leq t<\infty$ separately. We exemplify with the former. By Lemma 2.3,

$$
\begin{aligned}
\sup _{0<s \leq 1} \frac{\varrho(t s)}{\varrho(s)} & =\sup _{0<s \leq 1} \frac{\Psi(t s)}{\Psi(s)} \leq c(1+|\log t|)^{b} \\
\sup _{1 \leq s \leq t^{-1}} \frac{\varrho(t s)}{\varrho(s)} & =\sup _{1 \leq s \leq t^{-1}} \Psi(t s) \Psi\left(s^{-1}\right) \leq\left(\sup _{t \leq \sigma \leq 1} \Psi(\sigma)\right)^{2} \\
& \leq[\max (\Psi(t), \Psi(1))]^{2} \leq c^{2}(1+|\log t|)^{2 b}, \\
\sup _{t^{-1} \leq s<\infty} \frac{\varrho(t s)}{\varrho(s)} & =\sup _{t^{-1} \leq s<\infty} \frac{\Psi\left(s^{-1}\right)}{\Psi\left(t^{-1} s^{-1}\right)}=\sup _{0<\sigma \leq 1} \frac{\Psi(\sigma t)}{\Psi(\sigma)} \leq c(1+|\log t|)^{b} .
\end{aligned}
$$

On the other hand, also by Lemma 2.3, $\varrho(t) \geq \varrho(t) \geq c(1+|\log t|)^{-b}, \quad t \in(0, \infty)$.

Remark 2.6 From Lemma 2.5 we can infer that, for the function $\varrho$ in (2.7), the corresponding function $\bar{\varrho}$ has Boyd upper and lower indices $\alpha_{\bar{\varrho}}=\beta_{\bar{\varrho}}=0$.

Lemma 2.7 Let $g:(0, \infty) \rightarrow(0, \infty)$ be defined by

$$
g(t)=t^{\theta} \varrho\left(t^{\sigma}\right)^{\gamma}, \quad t \in(0, \infty),
$$

where $\theta, \sigma, \gamma \in \mathbb{R}$ and $\varrho$ is the function given by (2.7). Then $g$ belongs to the class $\mathcal{B}$ and the corresponding function $\bar{g}$ has Boyd upper and lower indices $\alpha_{\bar{g}}=\beta_{\bar{g}}=\theta$. 
Proof. The fact that $g$ belongs to the class $\mathcal{B}$ is a consequence of Lemma 2.5 and the properties stated in [15], namely Proposition 3 and Example 1 on pp. 184-185. Moreover, by straightforward calculations,

$$
\bar{g}(t)=t^{\theta} \bar{\varrho}\left(t^{\frac{|\gamma|}{\gamma} \sigma}\right)^{|\gamma|}, \quad t \in(0, \infty)
$$

and then, using Remark 2.6 as well as Proposition 2 and Example 1 of [15, pp. 184-185], we obtain

$$
\alpha_{\bar{g}}=\beta_{\bar{g}}=\theta \text {. }
$$

\subsection{Function spaces}

Since the definition of the Besov and Triebel-Lizorkin spaces $B_{p q}^{s}$ and $F_{p q}^{s}$ are by now quite standard (cf., for example, [23, pp. 49-50]), we will omit the corresponding details and just mention that the spaces $B_{p q}^{(s, \Psi)}$ and $F_{p q}^{(s, \Psi)}$ we want to consider here are obtained from the former by substituting the terms $2^{j s}, j \in \mathbb{N}_{0}$, controlling the smoothness, by the terms $2^{j s} \Psi\left(2^{-j}\right), j \in \mathbb{N}_{0}$, where $\Psi$ is an admissible function.

These spaces were introduced by Edmunds and Triebel in [3, 4] and also considered by Moura in [16], where they have remarked that such spaces are independent of the resolution of unity considered, in the sense of equivalent quasi-norms. If $\Psi \equiv 1$ then the spaces $B_{p q}^{(s, \Psi)}$ and $F_{p q}^{(s, \Psi)}$ clearly coincide with the usual Besov and Triebel-Lizorkin spaces, $B_{p q}^{s}$ and $F_{p q}^{s}$, respectively, and the following elementary embeddings hold:

$$
A_{p q}^{s+\varepsilon} \hookrightarrow A_{p q}^{(s, \Psi)} \hookrightarrow A_{p q}^{s-\varepsilon}
$$

for all $\varepsilon>0$ and $A \in\{B, F\}$. It is not difficult to see that for the results we intend to prove there is no loss of generality if we assume that the admissible function $\Psi$ is continuous and $\Psi(1)=1$, as there is always an equivalent such function. Therefore, from now on we suppose that this will be always the case, when such properties are technically important in a proof.

An important tool is the characterization of the spaces of generalized smoothness by means of atomic decompositions. We refer to [16] for a complete description. Here we need only that property in the case of spaces $B_{p q}^{(s, \Psi)}$ and will just point out the differences with respect to the well-known corresponding result for the spaces $B_{p q}^{s}$.

First, for the definition of an $(s, p, \Psi)_{K, L}$-atom we can rely on [23, Def. 13.3], where the corresponding condition (13.18) should now be replaced by

$$
\left|D^{\alpha} a(x)\right| \leq 2^{-\nu\left(s-\frac{n}{p}\right)+|\alpha| \nu} \Psi\left(2^{-\nu}\right)^{-1} .
$$

Then we have an atomic decomposition characterization for $B_{p q}^{(s, \Psi)}$ which can be stated almost in the same terms as in [23, Thm. 13.8 (i)]: one just has to change $(s, p, \Psi)_{K, L}$-atoms for $(s, p)_{K, L}$-atoms.

It will be important for us to realize that the spaces $B_{p q}^{(s, \Psi)}$ can be obtained from the usual Besov spaces by means of interpolation with a function parameter (cf. [15] and [2], for instance).

Let $s_{1}, s_{2} \in \mathbb{R}$ be such that $s_{1}<s<s_{2}$. Let $\Psi$ be a continuous admissible function with $\Psi(1)=1$. Then, by Theorem 13 of [15, p. 194] complemented by Theorem 5.3 and Remark 5.4 of [2, p. 166], we can write

$$
B_{p q}^{(s, \Psi)}=\left(B_{p 1}^{s_{1}}, B_{p 1}^{s_{2}}\right)_{g, q}
$$

where $g \in \mathcal{B}$ is the function parameter

$$
g(t)=t^{\left(s-s_{1}\right) /\left(s_{2}-s_{1}\right)} \varrho\left(t^{-1 /\left(s_{2}-s_{1}\right)}\right)
$$

and $\varrho$ is given by (2.7). We remark that Lemma 2.7 is important here, as it guarantees that

$$
\alpha_{\bar{g}}=\beta_{\bar{g}}=\frac{s-s_{1}}{s_{2}-s_{1}} \in(0,1) .
$$


Before finishing this subsection we shall recall the definition of the generalized Lorentz spaces $\Lambda^{q}(\phi)$. Following [15], if $\phi \in \mathcal{B}$ and $0<q \leq \infty$ it is the set of all complex measurable functions $f$ on $\mathbb{R}^{n}$ such that

$$
\left\|f \mid \Lambda^{q}(\phi)\right\|:= \begin{cases}\left(\int_{0}^{\infty}\left(\phi(t) f^{*}(t)\right)^{q} \frac{d t}{t}\right)^{1 / q}, & 0<q<\infty, \\ \sup _{t \in(0, \infty)} \phi(t) f^{*}(t), & q=\infty,\end{cases}
$$

is finite, where $f^{*}$ stands for the decreasing rearrangement of $f$. If $\phi(t)=t^{1 / p}(1+|\log t|)^{a}$ with $0<p \leq \infty$ and $a \in \mathbb{R}$, then $\Lambda^{q}(\phi)$ is the Lorentz-Zygmund space $L_{p q}(\log L)_{a}$, which in turn is the classical Lorentz space $L_{p q}$ if $a=0$.

The interpolation of Lebesgue spaces by a function parameter gives rise to function spaces in the latter scale. Let $0<p_{1}<p_{2} \leq \infty, 0<q \leq \infty$ and $g \in \mathcal{B}$ with $0<\beta_{\bar{g}} \leq \alpha_{\bar{g}}<1$. Applying Theorem 3 of [15],

$$
\left(L_{p_{1}}, L_{p_{2}}\right)_{g, q}=\Lambda^{q}(\gamma)
$$

where

$$
\gamma(t)=t^{1 / p_{1}} g\left(t^{\left(1 / p_{1}-1 / p_{2}\right)}\right)^{-1}
$$

\subsection{Local growth envelopes}

For the concept of growth envelope one should deal with function spaces of regular distributions (so, interpreted as functions in $L_{1}^{\text {loc }}$ ).

From the necessary and sufficient conditions for $B_{p q}^{s} \subset L_{1}^{\text {loc }}$ or $F_{p q}^{s} \subset L_{1}^{\text {loc }}$, cf. [20, Thm. 3.3.2, p. 114], and the embedding assertion (2.9), we can easily infer that the case $s<\sigma_{p}:=n(1 / p-1)_{+}$is out of consideration whereas $s>\sigma_{p}$ entails $A_{p q}^{(s, \Psi)} \subset L_{1}^{\text {loc }}$. Moreover, since the extremal situation $s=\sigma_{p}$ doesn't fit in the mainstream considerations in what follows we shall always assume that $s>\sigma_{p}$. From the known characterization of the parameters $s, p$ and $q$ that lead $F_{p q}^{s}$ and $B_{p q}^{s}$ to be continuously embedded in $L_{\infty}$ (see Introduction) and from the embeddings in (2.9) we immediately get that

$$
\text { if } s>\frac{n}{p} \text { then } A_{p q}^{(s, \Psi)} \hookrightarrow L_{\infty}, \quad \text { and } \quad \text { if } s<\frac{n}{p} \text { then } A_{p q}^{(s, \Psi)} \hookrightarrow L_{\infty} .
$$

We postpone to a subsequent work the study of the case $\sigma_{p}<s=n / p$ (called critical), for which different techniques are needed. In the case $\sigma_{p}<s<n / p$, which we want to study here (the so-called sub-critical case), we have just pointed out that we always have that $A_{p q}^{(s, \Psi)}$ is not continuously embedded in $L_{\infty}$, independently of the admitted $\Psi$ and $q$. We can even say, in such a case, that

$$
\mathcal{E}_{\mathrm{LG}} \mid A_{p q}^{(s, \Psi)}(t):=\sup \left\{f^{*}(t):\left\|f \mid A_{p q}^{(s, \Psi)}\right\| \leq 1\right\}
$$

defines a (finite) positive, decreasing function in $(0,1]$ which tends to $\infty$ as $t$ goes to 0 (the less trivial facts in this assertion can again be easily obtained by conjugating (2.9) with the corresponding assertions for $A_{p q}^{s}$ (cf. [25, Prop. 12.6])). Therefore, it makes sense to ask for the behaviour of $\mathcal{E}_{\mathrm{LG}} \mid A_{p q}^{(s, \Psi)}(t)$ near zero, which gives an indication of the ability of local growth for functions in $A_{p q}^{(s, \Psi)}$. This motivates the definition below, which tries to make more precise the corresponding idea of Haroske [8] and Triebel [25].

Definition 2.8 The local growth envelope function of $A_{p q}^{(s, \Psi)}$, for $\sigma_{p}<s<n / p$, is the equivalence class $\left[\mathcal{E}_{\mathrm{LG}} \mid A_{p q}^{(s, \Psi)}\right]$ of functions in $\mathcal{E}_{\mathrm{LG}}$. We shall also call local growth envelope function of $A_{p q}^{(s, \Psi)}$ any representative in such a class. We even call local growth envelope function of $A_{p q}^{(s, \Psi)}$ any function $f:(0, \varepsilon] \rightarrow \mathbb{R}^{+}$, for some $\varepsilon \in(0,1]$, even if not decreasing, such that $f \sim \mathcal{E}_{\mathrm{LG}} \mid A_{p q}^{(s, \Psi)}$ in $(0, \varepsilon]$, and use it to represent the equivalence class $\left[\mathcal{E}_{\mathrm{LG}} \mid A_{p q}^{(s, \Psi)}\right]$.

Let again $\sigma_{p}<s<n / p$.

Assume there exists a continuous representative $\mathcal{E}_{\mathrm{LG}} A_{p q}^{(s, \Psi)} \in\left[\mathcal{E}_{\mathrm{LG}} \mid A_{p q}^{(s, \Psi)}\right]$ (we shall later see that this is indeed the case). Let $(0, \varepsilon], 0<\varepsilon<1$, be its domain. 
Define $H(t):=-\log \mathcal{E}_{\mathrm{LG}} A_{p q}^{(s, \Psi)}(t)$ and note that $H$ is a (finite) real increasing function on $(0, \varepsilon]$ which tends to $-\infty$ when $t$ goes to 0 . There is only a Borel measure (i.e., a measure defined on the Borel sets) $\mu_{H}$ in $(0, \varepsilon]$ such that $\mu_{H}([a, b])=H(b)-H(a)$, for all $[a, b] \subset(0, \varepsilon]$. Its restriction to each such $[a, b]$ is the Stieltjes-Borel measure associated with $\left.H\right|_{[a, b]}$.

In the important case when $H$ happens to be continuously differentiable in $(0, \varepsilon]$, we have $\mu_{H}(d t)=H^{\prime} d t$, and for the functions we want to integrate we can calculate the integrals as improper Riemann integrals.

Definition 2.9 Let $\sigma_{p}<s<n / p$ and $0<u \leq \infty$. The couple

$$
\left(\left[\mathcal{E}_{\mathrm{LG}} \mid A_{p q}^{(s, \Psi)}\right], u\right)
$$

is called the local growth envelope of $A_{p q}^{(s, \Psi)}$ if $u$ is the minimum (assuming that it exists) of all $v>0$ such that

$$
\exists c(v)>0: \forall f \in A_{p q}^{(s, \Psi)}, \quad\left(\int_{(0, \varepsilon]}\left(h(t) f^{*}(t)\right)^{v} \mu_{H}(d t)\right)^{1 / v} \leq c(v)\left\|f \mid A_{p q}^{(s, \Psi)}\right\|,
$$

where $h(t)^{-1}$ is a continuous representative in $\left[\mathcal{E}_{\mathrm{LG}} \mid A_{p q}^{(s, \Psi)}\right]$ with domain $(0, \varepsilon], 0<\varepsilon<1$.

We must remark that this definition makes sense, namely that the infimum of all such $v$ 's is independent of the chosen continuous representative $h(t)^{-1}$ in $\left[\mathcal{E}_{\mathrm{LG}} \mid A_{p q}^{(s, \Psi)}\right]$, as follows by using some standard arguments of measure and integration theory, the definition of $\mathcal{E}_{\mathrm{LG}} \mid A_{p q}^{(s, \Psi)}$ and Proposition 12.2 (ii) of [25, p. 184]. Recall, on the other hand, that the definition of $\mathcal{E}_{\mathrm{LG}} \mid A_{p q}^{(s, \Psi)}$ guarantees that (2.13) holds at least for $v=\infty$.

Instead of $\left(\left[\mathcal{E}_{\mathrm{LG}} \mid A_{p q}^{(s, \Psi)}\right], u\right)$, we shall usually write $\left(h(t)^{-1}, u\right)$ for the local growth envelope of $A_{p q}^{(s, \Psi)}$ with $\sigma_{p}<s<n / p$, where $h(t)^{-1}$ is any continuous representative in $\left[\mathcal{E}_{\mathrm{LG}} \mid A_{p q}^{(s, \Psi)}\right]$. Instead of $h(t)^{-1}$, we can also use in the couple any local growth envelope function as considered in Definition 2.8, though it must be borne in mind that for the construction of the measure $\mu_{H}$ we shall only use continuous representatives in $\left[\mathcal{E}_{\mathrm{LG}} \mid A_{p q}^{(s, \Psi)}\right]$.

As to the interest in considering an estimate like (2.13), we notice that there is no point in trying to get that

$$
\exists c(v)>0: \forall f \in A_{p q}^{(s, \Psi)}, \quad\left(\int_{(0, \varepsilon]}\left(\chi(t) h(t) f^{*}(t)\right)^{v} \mu_{H}(d t)\right)^{1 / v} \leq c(v)\left\|f \mid A_{p q}^{(s, \Psi)}\right\|,
$$

with $\chi(t)$ a non-negative decreasing function in $(0, \varepsilon]$ such that $\lim _{t \rightarrow 0^{+}} \chi(t)=\infty$, simply because it doesn't exist such a function $\chi(t)$. This follows by the same type of arguments used in the proof of Proposition 12.10 in [25].

\section{A lifting property and embeddings}

\subsection{Lifting property}

Let $\left(\varphi_{j}\right)_{j=0}^{\infty}$ be a dyadic resolution of unity according to [23, (10.3), (10.4)], where $\varphi_{0} \in \mathcal{S}$ is now assumed to be non-negative and radially monotonically decreasing. Let $\Psi$ be an admissible function and define

$$
\widetilde{\Psi}(\xi):=\sum_{j=0}^{\infty} \Psi\left(2^{-j}\right) \varphi_{j}(\xi), \quad \xi \in \mathbb{R}^{n}
$$

Lemma 3.1 The functions $\widetilde{\Psi}$ and $1 / \widetilde{\Psi}$ belong to $C^{\infty}$. Moreover, for any $\alpha \in \mathbb{N}_{0}^{n}$ it holds

$$
\begin{aligned}
\left|\left(D^{\alpha} \widetilde{\Psi}\right)(\xi)\right| \leq c \Psi\left(\langle\xi\rangle^{-1}\right)\langle\xi\rangle^{-|\alpha|}, & \xi \in \mathbb{R}^{n}, \\
\left|\left(D^{\alpha}(1 / \widetilde{\Psi})\right)(\xi)\right| \leq c^{\prime} \Psi\left(\langle\xi\rangle^{-1}\right)^{-1}\langle\xi\rangle^{-|\alpha|}, & \xi \in \mathbb{R}^{n},
\end{aligned}
$$

where $\langle\xi\rangle:=\left(1+|\xi|^{2}\right)^{1 / 2}$, and the positive constants $c$, $c^{\prime}$ depend only on $\alpha$ and $\Psi$. 
Proof. Step 1. In this step we prove (3.2). Let $\alpha \in \mathbb{N}_{0}^{n}$ be fixed and let first $\xi \in \mathbb{R}^{n}$ be such that $2^{k-1} \leq$ $|\xi| \leq 2^{k+1}$, for some $k \in \mathbb{N}$ with $k \geq 2$. From (3.1) we have

$$
\left(D^{\alpha} \widetilde{\Psi}\right)(\xi)=\sum_{j=k-1}^{k+1} \Psi\left(2^{-j}\right) 2^{(-j+1)|\alpha|}\left(D^{\alpha} \varphi_{1}\right)\left(2^{-j+1} \xi\right) .
$$

Thus, using the fact that $\Psi\left(2^{-j}\right) \sim \Psi\left(2^{-(j+1)}\right), j \in \mathbb{N}$, the monotonicity of $\Psi$ and $\langle\xi\rangle \sim|\xi|$ for $|\xi| \geq 1$, we get

$$
\left|\left(D^{\alpha} \widetilde{\Psi}\right)(\xi)\right| \leq c_{1} \Psi\left(2^{-k}\right) 2^{-k|\alpha|} \leq c_{2} \Psi\left(\langle\xi\rangle^{-1}\right)\langle\xi\rangle^{-|\alpha|} .
$$

The remaining case, i.e. when $\xi \in \mathbb{R}^{n}$ is such that $|\xi| \leq 2$, is immediate.

Step 2. In this step we prove (3.3). First of all we remark that $\widetilde{\Psi}$ is a positive function. Let $\alpha \in \mathbb{N}_{0}^{n}$ with $|\alpha|=1$, i.e. $D^{\alpha}=\partial / \partial x_{i}$ for some $i \in\{1, \ldots, n\}$. We have

$$
\left(D^{\alpha}(1 / \widetilde{\Psi})\right)(\xi)=-\widetilde{\Psi}(\xi)^{-2}\left(D^{\alpha} \widetilde{\Psi}\right)(\xi)
$$

Assuming, without loss of generality, that $\xi \in \mathbb{R}^{n}$ is such that $2^{k-1} \leq|\xi| \leq 2^{k+1}$, for some $k \in \mathbb{N}$ with $k \geq 2$, we obtain

$$
\widetilde{\Psi}(\xi)=\sum_{j=k-1}^{k+1} \Psi\left(2^{-j}\right) \varphi_{j}(\xi) \geq c \Psi\left(2^{-k}\right) .
$$

Hence, from (3.4), (3.5), (3.2) and $\langle\xi\rangle \sim|\xi|$ for $|\xi| \geq 1$,

$$
\begin{aligned}
\left|\left(D^{\alpha}(1 / \widetilde{\Psi})\right)(\xi)\right| & \leq \widetilde{\Psi}(\xi)^{-2}\left|\left(D^{\alpha} \widetilde{\Psi}\right)(\xi)\right| \\
& \leq c_{1} \Psi\left(2^{-k}\right)^{-2} \Psi\left(\langle\xi\rangle^{-1}\right)\langle\xi\rangle^{-|\alpha|} \leq c_{2} \Psi\left(\langle\xi\rangle^{-1}\right)^{-1}\langle\xi\rangle^{-|\alpha|} .
\end{aligned}
$$

In this way we have shown (3.3) for $|\alpha|=1$. The case $|\alpha|=0$ can also be easily checked. The rest can be done by induction on $|\alpha|$.

Let $\Psi$ be an admissible function, according to Definition 2.1, and $\widetilde{\Psi}$ as in (3.1). Define $J^{\widetilde{\Psi}}$ by

$$
J^{\widetilde{\Psi}} f=(\widetilde{\Psi}(\cdot) \widehat{f})^{\vee}, \quad f \in \mathcal{S}^{\prime}
$$

Proposition 3.2 Let $\Psi$ be an admissible function according to Definition 2.1. Then $J^{\widetilde{\Psi}}$ is a topological isomorphism from

(i) $B_{p q}^{(s, \Psi)}$ onto $B_{p q}^{s}$, for any $0<p \leq \infty, 0<q \leq \infty$ and $s \in \mathbb{R}$,

(ii) $F_{p q}^{(s, \Psi)}$ onto $F_{p q}^{s}$, for any $0<p<\infty, 0<q \leq \infty$ and $s \in \mathbb{R}$.

Proof. We present only the proof of (ii) since the proof of (i) can be carried on by minor modifications. Having into consideration that for any $\sigma \in \mathbb{R}$ the map $I_{\sigma}$, given by

$$
I_{\sigma} f=\left(\langle\cdot\rangle^{\sigma} \widehat{f}\right)^{\vee}, \quad f \in \mathcal{S}^{\prime}
$$

defines an isomorphism between $F_{p q}^{(s, \Psi)}$ and $F_{p q}^{(s-\sigma, \Psi)}$, for any admitted parameters $s, p$ and $q$ (cf. [16, Prop. 1.8]), we can restrict the proof to the case $s=0$.

Step 1. Let $f \in F_{p q}^{(0, \Psi)}$. We have

$$
\begin{aligned}
\left\|J^{\widetilde{\Psi}} f \mid F_{p q}^{0}\right\| & =\|\|\left\{\left(\widetilde{\Psi} \varphi_{j} \widehat{f}\right)^{\vee}(\cdot)\right\}_{j=0}^{\infty}\left|\ell_{q}\left\|\mid L_{p}\right\|\right. \\
& =\|\|\left\{\left(\Psi\left(2^{-j}\right) M_{j} \varphi_{j} \widehat{f}\right)^{\vee}(\cdot)\right\}_{j=0}^{\infty}\left|\ell_{q}\left\|\mid L_{p}\right\|,\right.
\end{aligned}
$$


where

$$
M_{j}(x)=\Psi\left(2^{-j}\right)^{-1} \widetilde{\Psi}(x) h\left(2^{-j} x\right), \quad x \in \mathbb{R}^{n}, \quad j \in \mathbb{N},
$$

with $h \in \mathcal{S}$ such that

$$
h(x)=1 \text { if } 1 / 2 \leq|x| \leq 2 \text { and } \operatorname{supp} h \subset\left\{x \in \mathbb{R}^{n}: 1 / 4 \leq|x| \leq 4\right\}
$$

and

$$
M_{0}(x)=\Psi(1)^{-1} \widetilde{\Psi}(x) H(x), \quad x \in \mathbb{R}^{n}
$$

with $H \in \mathcal{S}$ such that

$$
H(x)=1 \text { if }|x| \leq 2 \text { and } \operatorname{supp} H \subset\left\{x \in \mathbb{R}^{n}:|x| \leq 4\right\}
$$

Let $\eta \in \mathbb{N}$ be such that $\eta>n / 2+n / \min (p, q)$. Using the formulae (3.2) from Lemma 3.1 and by straightforward calculations we can prove that

$$
\sup _{j \in \mathbb{N}_{0}}\left\|M_{j}\left(2^{j+2} \cdot\right) \mid W_{2}^{\eta}\right\|<\infty
$$

Hence, by (3.7) and applying Theorem 1.6.3 in [22], we obtain

$$
\begin{aligned}
\left\|J^{\widetilde{\Psi}} f \mid F_{p q}^{0}\right\| & \leq c_{1} \sup _{j \in \mathbb{N}_{0}}\left\|M _ { j } ( 2 ^ { j + 2 } \cdot ) \left|W _ { 2 } ^ { \eta } \| \| \left\|\{ ( \Psi ( 2 ^ { - j } ) \varphi _ { j } \widehat { f } ) ^ { \vee } ( \cdot ) \} _ { j = 0 } ^ { \infty } \left|\ell_{q}\left\|\mid L_{p}\right\|\right.\right.\right.\right. \\
& \leq c_{2}\left\|f \mid F_{p q}^{(0, \Psi)}\right\| .
\end{aligned}
$$

Step 2. Clearly $\left(J^{\widetilde{\Psi}}\right)^{-1}=J^{1 / \widetilde{\Psi}}$. The proof that it defines a bounded map from $F_{p q}^{0}$ into $F_{p q}^{(0, \Psi)}$ can be done analogously to the previous step, in this case using the formulae (3.3) from Lemma 3.1.

Remark 3.3 In [2, p. 162] there is a similar lifting property, but in this way we can avoid requiring further assumptions on the admissible function $\Psi$. In particular, we don't need the function $\Psi$ itself to be a $C^{\infty}$ function.

\subsection{Embeddings}

We use the lifting property from the previous subsection in order to transfer to spaces of generalized smoothness the well-known embeddings between the usual Besov and Triebel-Lizorkin spaces. In the following, $A_{p q}^{s}$ and $G_{p q}^{s}$ denote either a Besov or Triebel-Lizorkin space, $B_{p q}^{s}$ or $F_{p q}^{s}$. And a similar assumption corresponding to spaces of generalized smoothness, by inserting an admissible function $\Psi$.

Proposition 3.4 Let $\Psi$ be an admissible function and $s_{i}, p_{i}, q_{i}, i=1,2$, admitted parameters according to the definition of the spaces. Then the embedding

$$
A_{p_{1} q_{1}}^{s_{1}} \hookrightarrow G_{p_{2} q_{2}}^{s_{2}}
$$

holds if, and only if,

$$
A_{p_{1} q_{1}}^{\left(s_{1}, \Psi\right)} \hookrightarrow G_{p_{2} q_{2}}^{\left(s_{2}, \Psi\right)}
$$

holds.

Pr o of. We have the following commutative diagram 


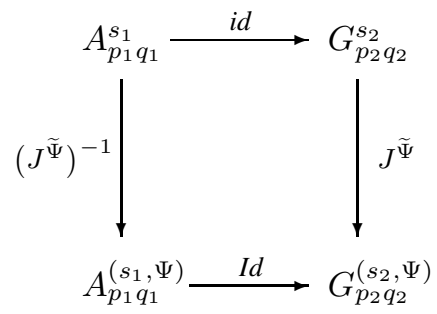

where $i d$ and $I d$ are the natural injections between the indicated spaces and $J^{\widetilde{\Psi}}$ is the operator in (3.6). Another commutative diagram is obtained by interchanging the vertical arrows. Then the assertion is a consequence of Proposition 3.2.

Example 3.5 In view of the embedding assertion in e.g. [23, 11.4, p. 55], we give an application of the last proposition. Let $\Psi$ be an admissible function according to Definition $2.1,0<p_{1}<p<p_{2} \leq \infty$ and suppose that

$$
s_{1}-\frac{n}{p_{1}}=s-\frac{n}{p}=s_{2}-\frac{n}{p_{2}}
$$

Then

$$
B_{p_{1} u}^{\left(s_{1}, \Psi\right)} \hookrightarrow F_{p q}^{(s, \Psi)} \hookrightarrow B_{p_{2} v}^{\left(s_{2}, \Psi\right)}
$$

if, and only if,

$$
0<u \leq p \leq v \leq \infty
$$

\section{Local growth envelopes for $B_{p q}^{(s, \Psi)}$ and $F_{p q}^{(s, \Psi)}$ in the sub-critical case}

We noticed before (see Subsection 2.2) that for the results we have in mind there is no loss of generality in considering only admissible functions $\Psi$ which are continuous and such that $\Psi(1)=1$. We shall stick to this in the proofs that follow. Recall that $\sigma_{p}:=n(1 / p-1)_{+}$.

Proposition 4.1 Let $0<p, q \leq \infty$ and $s \in \mathbb{R}$ be such that $\sigma_{p}<s<n / p$. Let $\Psi$ be an admissible function and define $r \in(1, \infty)$ by the equation $s-n / p=-n / r$. Then there exists $c>0$ such that

$$
\mathcal{E}_{\mathrm{LG}} \mid B_{p q}^{(s, \Psi)}(t) \leq c t^{-1 / r} \Psi(t)^{-1}, \quad \text { for all } t \in(0,1]
$$

and, for each $v \in[q, \infty]$, there exists $c(v)>0$ such that

$$
\left(\int_{0}^{1}\left(t^{1 / r} \Psi(t) f^{*}(t)\right)^{v} \frac{d t}{t}\right)^{1 / v} \leq c(v)\left\|f \mid B_{p q}^{(s, \Psi)}\right\|, \text { for all } f \in B_{p q}^{(s, \Psi)}
$$

(with the modification (4.6) if $v=\infty$ ).

Proof. Consider $0<v \leq \infty$. By (2.10),

$$
B_{p v}^{(s, \Psi)}=\left(B_{p 1}^{s_{1}}, B_{p 1}^{s_{2}}\right)_{g, v},
$$

where $g \in \mathcal{B}$ is the function parameter

$$
g(t)=t^{\left(s-s_{1}\right) /\left(s_{2}-s_{1}\right)} \varrho\left(t^{-1 /\left(s_{2}-s_{1}\right)}\right)
$$

with $\varrho$ given by (2.7) and $s_{1}, s_{2} \in \mathbb{R}$ chosen in such a way that $s_{1}<s<s_{2}$. In the case $\sigma_{p}<s<n / p$, such $s_{1}$ and $s_{2}$ can be chosen in such a way that $r_{1}$ and $r_{2}$ defined by the equations $s_{i}-n / p=-n / r_{i}, i=1,2$, satisfy $r_{i}>p$ and $1<r_{i}<\infty, i=1,2$, so that

$$
B_{p 1}^{s_{1}} \hookrightarrow L_{r_{1}} \quad \text { and } \quad B_{p 1}^{s_{2}} \hookrightarrow L_{r_{2}}
$$


(cf. [23, 11.4 (iii) and 10.5 (i)]). Since, by Lemma 2.7 and (2.12),

$$
\left(L_{r_{1}}, L_{r_{2}}\right)_{g, v}=\Lambda^{v}(\gamma)
$$

with

$$
\gamma(t)=t^{1 / r_{1}} g\left(t^{1 / r_{1}-1 / r_{2}}\right)^{-1}=t^{1 / r} \varrho\left(t^{-1 / n}\right)^{-1},
$$

then this together with (4.3) and (4.4) imply that

$$
B_{p v}^{(s, \Psi)} \hookrightarrow \Lambda^{v}(\gamma)
$$

Using the definition of $\varrho, \Lambda^{v}(\gamma)$ and the fact that $\Psi\left(t^{1 / n}\right) \sim \Psi(t), t \in(0,1]$, (4.5) guarantees the existence of $c_{1}(v)>0$ such that

$$
\left(\int_{0}^{1}\left(t^{1 / r} \Psi(t) f^{*}(t)\right)^{v} \frac{d t}{t}\right)^{1 / v} \leq c_{1}(v)\left\|f \mid B_{p v}^{(s, \Psi)}\right\|, \quad \text { for all } \quad f \in B_{p v}^{(s, \Psi)} .
$$

Considering now $v$ restricted to $v \geq q$, elementary embeddings (cf. Proposition 3.4 and [22, 2.3.2/(5)]) guarantee the existence of $c_{2}(v)>0$ such that

$$
\begin{array}{r}
\left(\int_{0}^{1}\left(t^{1 / r} \Psi(t) f^{*}(t)\right)^{v} \frac{d t}{t}\right)^{1 / v} \leq c_{1}(v)\left\|f\left|B_{p v}^{(s, \Psi)}\left\|\leq c_{2}(v)\right\| f\right| B_{p q}^{(s, \Psi)}\right\|, \\
\text { for all } f \in B_{p q}^{(s, \Psi)} .
\end{array}
$$

This proves (4.2). Formula (4.1) follows easily by considering just the particular case of $v=\infty$ in the above expression, that is,

$$
\sup _{t \in(0,1]} t^{1 / r} \Psi(t) f^{*}(t) \leq c_{2}(\infty)\left\|f \mid B_{p q}^{(s, \Psi)}\right\|, \quad \text { for all } \quad f \in B_{p q}^{(s, \Psi)} .
$$

Proposition 4.2 Let $0<p, q \leq \infty$ and let $s \in \mathbb{R}$ be such that $\sigma_{p}<s<n / p$. Let $\Psi$ be an admissible function and define $r \in(1, \infty)$ by the equation $s-n / p=-n / r$. Then there exist $\varepsilon, c>0$ with $\varepsilon<1$ such that

$$
\mathcal{E}_{\mathrm{LG}} \mid B_{p q}^{(s, \Psi)}(t) \geq c t^{-1 / r} \Psi(t)^{-1}, \quad \text { for all } t \in(0, \varepsilon]
$$

and for each $v \in(0, q)$ there is no $c(v)>0$ such that

$$
\left(\int_{0}^{\varepsilon}\left(t^{1 / r} \Psi(t) f^{*}(t)\right)^{v} \frac{d t}{t}\right)^{1 / v} \leq c(v)\left\|f \mid B_{p q}^{(s, \Psi)}\right\|, \quad \text { for all } \quad f \in B_{p q}^{(s, \Psi)},
$$

can hold.

Pr o of. We follow closely the arguments of $[25,15.2]$, with natural modifications. For each $j \in \mathbb{N}$, consider $f_{j}$ given by

$$
f_{j}(x):=2^{j n / r} \Psi\left(2^{-j}\right)^{-1} \Phi\left(2^{j} x\right), \quad x \in \mathbb{R}^{n},
$$

where $\Phi$ is given by

$$
\Phi(x)=e^{-\frac{1}{1-|x|^{2}}} \text { if }|x|<1 \text { and } \Phi(x)=0 \text { if }|x| \geq 1
$$

Since $s>\sigma_{p}, f_{j}$ is, up to a constant factor, an atom for the space $B_{p q}^{(s, \Psi)}$, cf. [16, Def. 1.14 (ii) and Thm. 1.18 (ii)], more precisely, a $(s, p, \Psi)_{K,-1}$-atom, where $K>s$ is a fixed natural number. It is easily seen that

$$
f_{j}^{*}\left(d 2^{-j n}\right) \sim 2^{j n / r} \Psi\left(2^{-j}\right)^{-1}
$$


for some $d>0$ depending only on the function $\Phi$. On the other hand, it follows from the definition of $\mathcal{E}_{\mathrm{LG}} \mid B_{p q}^{(s, \Psi)}$ and Theorem 1.18 of [16] that

$$
\mathcal{E}_{\mathrm{LG}} \mid B_{p q}^{(s, \Psi)}\left(d 2^{-j n}\right) \geq c_{1} f_{j}^{*}\left(d 2^{-j n}\right), \text { for large enough natural } j,
$$

for some $c_{1}>0$. Therefore,

$$
\mathcal{E}_{\mathrm{LG}} \mid B_{p q}^{(s, \Psi)}\left(d 2^{-j n}\right) \geq c_{2} 2^{j n / r} \Psi\left(2^{-j}\right)^{-1}, \text { for large enough natural } j,
$$

for some $c_{2}>0$, which implies (4.7) (observe that $\Psi\left(t^{1 / n}\right) \sim \Psi(t)$ for $\left.t \in(0,1]\right)$.

Assume now that for some $v \in(0, q)$ it was possible to find $c(v)$ such that (4.8) holds. Consider, for each $J \in \mathbb{N}, f \in B_{p q}^{(s, \Psi)}$ defined by

$$
f(x)=\sum_{j=1}^{J} 2^{j n / r} \Psi\left(2^{-j}\right)^{-1} \Phi\left(2^{j} x-x^{0}\right),
$$

where $\Phi$ has the same meaning as before and $x^{0} \in \mathbb{Z}^{n}$ is chosen in such a way that the supports of $\Phi\left(2^{j} \cdot-x^{0}\right)$, $j \in \mathbb{N}$, are disjoint (it is enough to impose $\left|x^{0}\right|>3$, for this to be true). For $k=1, \ldots, J$ one has, for some $a, b>0$ depending only on $\Phi$, that

$$
\left|\left\{x \in \mathbb{R}^{n}:|f(x)|>b 2^{k n / r} \Psi\left(2^{-k}\right)^{-1}\right\}\right| \geq 2 a 2^{-k n} .
$$

Therefore,

$$
f^{*}\left(a 2^{-k n}\right) \geq b 2^{k n / r} \Psi\left(2^{-k}\right)^{-1}, \quad k=1, \ldots, J
$$

and we could write, for $k_{0}$ such that $a 2^{-k_{0} n} \leq \varepsilon$ (so, depending only on $\Phi, n$ and $\varepsilon$ ) and any $J \geq k_{0}$,

$$
\begin{aligned}
\left\|f \mid B_{p q}^{(s, \Psi)}\right\| & \geq c(v)^{-1}\left(\int_{0}^{\varepsilon}\left(t^{1 / r} \Psi(t) f^{*}(t)\right)^{v} \frac{d t}{t}\right)^{1 / v} \\
& \geq c(v)^{-1}\left(\sum_{k=k_{0}}^{\infty} \int_{a 2^{-(k+1) n}}^{a 2^{-k n}}\left(t^{1 / r} \Psi(t) f^{*}(t)\right)^{v} \frac{d t}{t}\right)^{1 / v} \\
& \geq c_{1}(v)\left(\sum_{k=k_{0}}^{J} b^{v}\right)^{1 / v} \\
& =c_{2}(v)\left(J-k_{0}+1\right)^{1 / v}
\end{aligned}
$$

where we have used Lemma 2.3 (ii), the monotonicity of $f^{*}$ and (4.10). On the other hand, and since (4.9) is an atomic decomposition in $B_{p q}^{(s, \Psi)}$, by Theorem 1.18 (ii) of [16] there is a constant $c_{4}>0$ such that

$$
\left\|f \mid B_{p q}^{(s, \Psi)}\right\| \leq c_{4}\left(\sum_{j=1}^{J} c_{3}^{-q}\right)^{1 / q}=c_{4} c_{3}^{-1} J^{1 / q}, \quad \text { for all } \quad J \in \mathbb{N} .
$$

Putting this together with (4.11), we get that

$$
\left(J-k_{0}+1\right)^{1 / v} \leq c_{3}(v) J^{1 / q}, \quad \text { for all } J \geq k_{0},
$$

which is clearly impossible when $v \in(0, q)$.

Corollary 4.3 Let $0<p, q \leq \infty$ and let $s \in \mathbb{R}$ be such that $\sigma_{p}<s<n / p$. Let $\Psi$ be an admissible function and define $r \in(1, \infty)$ by the equation $s-n / p=-n / r$. Then, for the same $\varepsilon \in(0,1)$ of the preceding 
proposition, there exists a continuously differentiable, positive and increasing function $h$ defined in $(0, \varepsilon]$ such that

$$
h(t)^{-1} \sim t^{-1 / r} \Psi(t)^{-1} \quad \text { in } \quad(0, \varepsilon]
$$

and satisfying

$$
0<\inf _{t \in(0, \varepsilon]} \frac{t h^{\prime}(t)}{h(t)} \leq \sup _{t \in(0, \varepsilon]} \frac{t h^{\prime}(t)}{h(t)}<\infty .
$$

Moreover, denoting by $\mu_{H}$ the Borel measure associated with $H:=\log h$ in $(0, \varepsilon]$ (in accordance with Section 2.3), and given any $v \in(0, \infty]$, there exists $c(v)>0$ such that

$$
\left(\int_{(0, \varepsilon]}\left(h(t) f^{*}(t)\right)^{v} \mu_{H}(d t)\right)^{1 / v} \leq c(v)\left\|f \mid B_{p q}^{(s, \Psi)}\right\|, \quad \text { for all } f \in B_{p q}^{(s, \Psi)},
$$

if, and only if, $v \geq q$.

Proof. Consider the function $\varrho \in \mathcal{B}$ as in (2.7) and the function $g:(0, \infty) \rightarrow(0, \infty)$ given by $g(t)=$ $t^{1 / r} \varrho(t)$. By Lemma 2.7, this function belongs to $\mathcal{B}$ and the corresponding function $\bar{g}$ (given as in (2.6)) has upper and lower Boyd indices $\alpha_{\bar{g}}$ and $\beta_{\bar{g}}$ equal to $1 / r>0$. By Def. 2 and Prop. 4 of [15, p. 185], we can then guarantee that there exists an increasing function $h \in \mathcal{B}$ with $h \sim g$ which is a $C^{1}$-diffeomorphism of $(0, \infty)$ and satisfies (4.13). In particular,

$$
\frac{h^{\prime}(t)}{h(t)} \sim \frac{1}{t}, \quad t \in(0, \infty),
$$

and $h(t)^{-1} \sim t^{-1 / r} \Psi(t)^{-1}$ in $(0, \varepsilon]$. We consider in what follows that $h$ has been restricted to this interval.

The two previous propositions, namely formulae (4.1) and (4.7), guarantee now that $h(t)^{-1}$ is a continuous representative in $\left[\mathcal{E}_{\mathrm{LG}} \mid B_{p q}^{(s, \Psi)}\right]$, and therefore we can consider the Borel measure $\mu_{H}$ associated with $H(t):=$ $\log h(t)$ in $(0, \varepsilon]$, cf. Section 2.3 . Since $h$ is continuously differentiable in $(0, \varepsilon]$, then

$$
\mu_{H}(d t)=\frac{h^{\prime}(t)}{h(t)} d t
$$

so that, due to (4.12) and (4.15), the left-hand sides of (4.8) and (4.14) coincide, up to a positive factor independent of $f \in B_{p q}^{(s, \Psi)}$. Then the assertion concerning (4.14) follows from the second halves of Proposition 4.1 and Proposition 4.2.

With this corollary we reached a point where the proof of the first assertion in the next result should be clear (recall also the conventions made in Section 2.3).

Theorem 4.4 Let $0<p, q \leq \infty$ and let $s \in \mathbb{R}$ be such that $\sigma_{p}<s<n / p$. Let $\Psi$ be an admissible function and define $r \in(1, \infty)$ by the equation $s-n / p=-n / r$. Then

(i) the local growth envelope of $B_{p q}^{(s, \Psi)}$ is $\left(t^{-1 / r} \Psi(t)^{-1}, q\right)$;

(ii) the local growth envelope of $F_{p q}^{(s, \Psi)}$ is $\left(t^{-1 / r} \Psi(t)^{-1}, p\right)$.

Pro of. It only remains to prove (ii). It is clearly possible to choose $\sigma_{p}<s_{1}, s_{2}<n / p$ and $0<p_{1}, p_{2}<\infty$ such that

$$
p_{1}<p<p_{2}, \quad s_{1}-\frac{n}{p_{1}}=s-\frac{n}{p}=s_{2}-\frac{n}{p_{2}}
$$

and $s_{1}>\sigma_{p_{1}}, s_{2}>\sigma_{p_{2}}$, so that the corollary above holds true both for $B_{p_{1} p}^{\left(s_{1}, \Psi\right)}$ and $B_{p_{2} p}^{\left(s_{2}, \Psi\right)}$. We can then apply Example 3.5 with $u=v=p$ to get that there exist $c_{1}, c_{2}>0$ such that

$$
h(t)^{-1} \sim_{\mathrm{LG}} c_{1} \mathcal{E}_{\mathrm{LG}}\left|B_{p_{1} p}^{\left(s_{1}, \Psi\right)}(t) \leq \mathcal{E}_{\mathrm{LG}}\right| F_{p q}^{(s, \Psi)}(t) \leq c_{2} \mathcal{E}_{\mathrm{LG}} \mid B_{p_{2} p}^{\left(s_{2}, \Psi\right)}(t) \sim_{\mathrm{LG}} h(t)^{-1},
$$

with $h$ given as in the above corollary.

Also, considering (4.14) for $B_{p_{1} p}^{\left(s_{1}, \Psi\right)}$ and $B_{p_{2} p}^{\left(s_{2}, \Psi\right)}$ instead of $B_{p q}^{(s, \Psi)}$, and using the embeddings of Example 3.5 in the case $u=v=p$, we obtain the required conclusion. 
We can interpret the assertion (ii) in the above theorem in the spirit considered before for the spaces $B_{p q}^{(s, \Psi)}$, as in Corollary 4.3. Thus, for some $\varepsilon \in(0,1)$, there exists a continuously differentiable, positive and increasing function $h$ defined in $(0, \varepsilon]$ such that

$$
\mathcal{E}_{\mathrm{LG}} \mid F_{p q}^{(s, \Psi)}(t) \sim h(t)^{-1} \sim t^{-1 / r} \Psi(t)^{-1} \quad \text { in } \quad(0, \varepsilon]
$$

and satisfying (4.13). With $H:=\log h$ and denoting by $\mu_{H}$ the Borel measure associated with $H$ in $(0, \varepsilon]$, and given any $v \in(0, \infty]$, there exists $c(v)>0$ such that

$$
\left(\int_{(0, \varepsilon]}\left(h(t) f^{*}(t)\right)^{v} \mu_{H}(d t)\right)^{1 / v} \leq c(v)\left\|f \mid F_{p q}^{(s, \Psi)}\right\|, \quad \text { for all } \quad f \in F_{p q}^{(s, \Psi)}
$$

if, and only if, $v \geq p$.

As before, instead of the left-hand side of (4.16) we can also write

$$
\left(\int_{0}^{\varepsilon}\left(t^{1 / r} \Psi(t) f^{*}(t)\right)^{v} \frac{d t}{t}\right)^{1 / v}
$$

Acknowledgements We take this opportunity to thank Professor Goldman and Dr. Haroske for valuable comments.

This research has been partially supported by Instituto de Cooperação Científica e Tecnológica Internacional (convénio ICCTI/DAAD). The first author has also been supported by Unidade de Investigação em Matemática e Aplicações da Universidade de Aveiro and the second author by Centro de Matemática da Universidade de Coimbra.

\section{References}

[1] Y. A. Brudnyi, On the rearrangement of a smooth function, Usp. Mat. Nauk 27, 165-166 (1972) (in Russian).

[2] F. Cobos and D. Fernandez, Hardy-Sobolev spaces and Besov spaces with a function parameter, Lect. Notes Math. 1302 (Springer-Verlag, 1988), pp. 158-170.

[3] D. E. Edmunds and H. Triebel, Spectral theory for isotropic fractal drums, C. R. Acad. Sci. Paris, Sér. I, Math. 326, 1269-1274 (1998).

[4] D. E. Edmunds and H. Triebel, Eigenfrequencies of isotropic fractal drums, Oper. Theory: Adv. Appl. 110, 81-102 (1999).

[5] M. L. Gold'man, Embedding of generalized Nikol'skij-Besov spaces in Lorentz spaces, Tr. Mat. Inst. Steklova 172, 128-139 (1985) (in Russian).

[6] M. L. Gold'man, On imbedding of constructive and structural Lipschitz spaces in symmetric spaces, Tr. Mat. Inst. Steklova 173, 90-112 (1986) (in Russian). English translation: Proc. Steklov Inst. Math. 4, 93-118 (1987).

[7] M. L. Goldman, Hardy type inequalities on the cone of quasimonotone functions, Tech. Report 98/31, Russian Academy of Sciences Far-Eastern Branch, Computer Center (Khabarovsk, 1998).

[8] D. D. Haroske, Envelopes in function spaces - a first approach, preprint Math/Inf/16/01 (Univ. Jena, Germany, 2001).

[9] C. Herz, Lipschitz spaces and Bernstein's theorem on absolutely convergent Fourier transforms, J. Math. Mech. 18, 283-324 (1968).

[10] G. A. Kalyabin, Criteria of the multiplication property and the embedding in C of spaces of Besov-Lizorkin-Triebel type, Mat. Zametki 30, 517-526 (1981) (in Russian).

[11] G. A. Kalyabin and P. I. Lizorkin, Spaces of generalized smoothness, Math. Nachr. 133, 7-32 (1987).

[12] V. I. Kolyada, Rearrangement of functions and embeddings of anisotropic spaces of Sobolev type, East J. Approx. 4, No. 2, 111-199 (1998).

[13] H.-G. Leopold, Embeddings and entropy numbers in Besov spaces of generalized smoothness, in: Function Spaces: The fifth Conference", edited by H. Hudzig and L. Skrzypczak, Lect. Notes Pure Appl. Math. 213 (Marcel Dekker, 2000), pp. 323-336.

[14] P. I. Lizorkin, Spaces of generalized smoothness, Appendix to the Russian edition of: H. Triebel, Theory of Function Spaces (Birkhäuser, Basel, 1983), (Mir, Moscow, 1986), pp. 381-416 (in Russian).

[15] C. Merucci, Applications of interpolation with a function parameter to Lorentz, Sobolev and Besov spaces, Lect. Notes Math. 1070 (Springer-Verlag, 1984), pp. 183-201.

[16] S. Moura, Function spaces of generalised smoothness, Diss. Math. 398, 1-88 (2001).

[17] Yu. Netrusov, Theorems of imbedding Besov spaces into ideal spaces, Zap. Nauchn. Semin. Leningr. Otd. Mat. Inst. Steklova 159, 69-82 (1987) (in Russian). English translation: J. Sov. Math. 47, 2871-2881 (1989). 
[18] Yu. Netrusov, Sets of singularities of functions in spaces of Besov and Lizorkin-Triebel type, Tr. Mat. Inst. Steklova 187, 162-177 (1989) (in Russian). English translation: Proc. Steklov Inst. Math. 187, 185-203 (1990).

[19] J. Peetre, Espaces d'interpolation et théorème de Soboleff, Ann. Inst. Fourier 16, 279-317 (1966).

[20] W. Sickel and H. Triebel, Hölder inequalitites and sharp embeddings in function spaces of $B_{p q}^{s}$ and $F_{p q}^{s}$ type, Z. Anal. Anwend. 14, No. 1, 105-140 (1995).

[21] R. S. Strichartz, Multipliers on fractional Sobolev spaces, J. Math. Mech. 16, 1031-1060 (1967).

[22] H. Triebel, Theory of Function Spaces (Birkhäuser, Basel, 1983).

[23] H. Triebel, Fractals and Spectra (Birkhäuser, Basel, 1997).

[24] H. Triebel, Sharp Sobolev embeddings and related Hardy inequalities: the sub-critical case, Math. Nachr. 208, 167-178 (1999).

[25] H. Triebel, The Structure of Functions (Birkhäuser, Basel, 2001). 